\title{
Linking low docosahexaenoic acid intake to Alzheimer's disease: caution recommended
}

\author{
Stephen C. CUNNANE \\ Mélanie PLOURDE \\ Milène VANDAL \\ Erika FREEMANTLE \\ Jennifer TREMBLAY-MERCIER \\ Michel BÉGIN
}

\section{Research Center on Aging, Health and Social}

Sciences Center, University Institute of Geriatrics of Sherbrooke, Université de Sherbrooke,

1036 Belvédère St. South, Sherbrooke, QC, Canada J1H 4C4

<Stephen.cunnane@usherbrooke.ca>

\section{Introduction}

As the percentage of elderly people in developed countries increases, awareness is growing rapidly about the pivotal importance of maintaining physical and cognitive autonomy for healthy aging. Alzheimer's disease (AD) represents the main form of cognitive decline in the adults. As such, preventing or avoiding the main risk factors for $A D$ improves the chances of prolonging cognitive function and quality of life in the elderly. Great efforts are now being made to understand the etiology of $A D$ and to develop strategies to attenuate its significant medical, social and economic impact. Currently, most treatment strategies for $A D$ focus on pharmacological interventions while most strategies for prevention focus on lifestyle risk factors, especially nutrition. According to a recent European report [1], no single strategy has been particularly successful at reducing the incidence or growing socio-economic impact

\footnotetext{
Abbreviations:

$A A$, arachidonic acid

$A D$, Alzheimer's disease

DHA, docosahexaenoic acid

$E P A$, eicosapentaenoic acid

$M C l$, mild cognitive impairment

MMSE, mini-mental state exam

$P E$, phosphatidylethanolamine
}

\begin{abstract}
Prospective cohort studies and animal models support the concept that low docosahexaenoic acid intake is implicated in the etiology or progression of Alzheimer's disease. However, other studies crucial to this relationship are less encouraging. To date, the few trials using docosahexaenoic acid to treat declining cognition in the elderly have either been very small or, in the largest trial, the beneficial effect was mild and limited to a sub-group of patients. The supplements used in each of these clinical trials contained at least one polyunsaturated fatty acid other than docosahexaenoic acid, so the active ingredient remains unclear. One widely cited study reported markedly lower brain docosahexaenoic acid in Alzheimer's disease but at least five other much less commonly cited reports have not corroborated this effect. There are numerous inconsistencies or confounders in the data and several challenges to overcome before definitively attributing a specific role to docosahexaenoic acid in the protection of cognitive function in the elderly.
\end{abstract}

Key words: docosahexaenoic acid, Alzheimer's disease, omega 3 fatty acids, brain, eicosapentaenoic acid, arachidonic acid

of $A D$ or other adult forms of significant cognitive decline.

There is growing concern that once $A D$ is clinically diagnosed it is already too late to initiate effective treatment. Hence, there is a burgeoning interest in nutritional approaches to reduce the risk of cognitive decline before it becomes clinically symptomatic. Amongst the various nutritional approaches, one that is gaining a lot of attention at the moment relates to low intake of fish and omega $(\omega) 3$ polyunsaturated fatty acids as a risk factor for $A D$. The $\omega 3$ fatty acid most studied in the context of $A D$ is docosahexaenoic acid (DHA; 22:603), which is found principally in fish and seafood. A possible link between dietary DHA and maintenance of brain function in adults has its origins in the study of infant brain development where DHA is now clearly recognized by most pediatric societies and government regulatory agencies as an essential component of optimal postnatal feeding practice.

At present, we are aware of four reports describing positive but very mild effects of a dietary DHA supplement in AD or other forms of cognitive deterioration in the elderly. In addition, one report showing lower brain DHA levels in AD is frequently cited. These studies compliment a much larger epidemiological literature linking low $\omega 3$ fatty acid and/or low DHA intake to higher risk of $A D$, a literature supported by several animal studies (which will not be reviewed here). Despite the apparent link between low $D H A$ intake and $A D$, significant confounders limit interpretation of the studies reporting a clinical benefit of DHA in $A D$. Several detailed reports of brain fatty acid data in $A D$ actually show no specific change or even a rise in brain DHA, but are not commonly cited. Hence, in the interests of encouraging a more critical perspective on the putative link between $D H A$ and $A D$, we provide here a brief review of the literature on clinical trials and brain $\mathrm{DHA}$ data in $\mathrm{AD}$.

\section{Linking low DHA and Alzheimer's disease}

A plausible association between DHA and risk reduction for cognitive decline in the elderly is emerging on three main fronts - epidemiological studies, plasma DHA levels in AD patients, and animal models. A task force of the International Association of Nutrition and Aging recently reviewed the literature on nutritional factors associated with aging-related cognitive decline [2]. This report provided details of 15 prospective studies conducted between 1997-2006 that focused on whether the risk of cognitive decline during aging is associated with low intake of total dietary fat, fish, and/or 133 fatty acids from fish. Collectively, these studies followed up an average of 40,000 people over 6 years. Two of the largest of these studies reported no significant association between $\omega 3$ or fish intake, but the others 
all showed a protective effect of fish and/or DHA on risk of cognitive decline. Other reviews [3] and a more recent epidemiological study [4] also came to the same conclusion.

Because of the normally strong positive association between plasma DHA and DHA or fish intake, it would be expected that a link between low fish or DHA intake and cognitive decline in the elderly would be reflected by lower plasma DHA in those experiencing cognitive decline. Indeed, several studies have found low plasma DHA in AD [5-8], but one study found that $A D$ was accompanied by higher plasma DHA [9].

\section{Clinical efficacy of DHA in Alzheimer's disease}

Regardless of the plausibility of a relationship that emerges from other types of studies, clearly, the central issue in linking low DHA to risk of $A D$ is whether higher $D H A$ intake has a lasting clinical beneficial effect in preventing or reducing cognitive decline in any form of neurodegeneration, most particularly in AD. No other type of study can supplant the importance of a series of independently run, welldesigned clinical interventions that collectively support a therapeutic benefit of DHA. In the largest study to date, Freund-Levi et al. [10] recently reported the results of a randomized, placebo-controlled, double blind trial, in which a mixed supplement containing both DHA $(1.7 \mathrm{~g} / \mathrm{d})$ and eicosapentaenoic acid (EPA - 20:503; $0.6 \mathrm{~g} / \mathrm{d}$ ) was given to patients with mild to moderate AD. The patients continued to receive their usual medication and received the active treatment $(\mathrm{DHA}+\mathrm{EPA})$ or placebo for 6 months followed by active treatment of both groups for a further 6 months. In the 174 participants completing the $12 \mathrm{mo}$ trial, no difference in cognitive function between the two groups was observed after the first 6 months. In a small sub-group with relatively high cognitive function at the start of the study, cognitive scores (mini-mental state exam; MMSE) and delayed word recall declined more slowly on the active treatment. More rapid decline of the MMSE score during the first 6 months in those with a starting MMSE of $<24$ was noted but was not statistically significant.

Three smaller trials using DHA in AD [11-13] have been reported but two of these studies appear to have only been published in preliminary form [11, 12]. Terano et al. [11] randomized 20 elderly vascular dementia patients in a nursing home to receive no treatment or a DHA supplement ( $4.3 \mathrm{~g} / \mathrm{d}$ for $12 \mathrm{mo}$ ). Initially, these patients all had cognitive evaluations consistent with mild to moderate dementia. Depending on the cognitive test used, improvement in the DHA group was seen at 3 and/or $6 \mathrm{mo}$, but not at 12 mo. Suzuki et al. [12] evaluated the effect of $680-800 \mathrm{mg} / \mathrm{d}$ of DHA in 22 elderly patients with cognitive decline of mixed etiology. In about half the patients, some cognitive parameters improved during the 6 month trial. Kotani et al. [13] evaluated the effect of a $90 \mathrm{~d}$ treatment using a mixture providing a total of $240 \mathrm{mg} / \mathrm{d}$ of DHA and arachidonic acid (AA; 20:406) in patients with AD or mild cognitive impairment $(\mathrm{MCl})$. The proportion of DHA to $A A$ in the mixture was not given. $A D$ patients $(n=8)$ only received the active treatment. $M C$ patients received either the active treatment $(\mathrm{DHA}+\mathrm{AA} ; \mathrm{n}=12)$ or a placebo ( 6 capsules of olive oil providing $240 \mathrm{mg} / \mathrm{d} ; \mathrm{n}=9$ ). Attention and immediate memory scores improved in the $\mathrm{MCl}$ group given $\mathrm{DHA}+\mathrm{AA}$ but not in the other groups.

Boston et al. [14] reported that there was no effect of a 12 wk treatment with $1 \mathrm{~g} / \mathrm{d}$ ethyl-EPA on several parameters of cognition in $A D$ patients who had a starting MMSE score of 19. This report is noted here because it used one of the fish oil $\omega 3$ fatty acids (EPA) but this highly purified preparation contained no DHA.

These clinical studies highlight several challenges associated with investigating a beneficial effect of $\omega 3$ fatty acids in AD. In all four of these trials [10-13], more than one potentially active polyunsaturate was present, so the question arises as to whether the beneficial effect due to DHA alone, or to the EPA [10-12] or AA [13] also provided with the $D H A$, or to the combinations of $\mathrm{DHA}+\mathrm{EPA}$ or $\mathrm{DHA}+\mathrm{AA}$. Assuming that half the active treatment in the study by Kotani et al. [13] was DHA, what explains how their results appear to be broadly equivalent to those of Freund-Levi et al. [10] who provided 14 times as much DHA, or those of Terano et al. [11], who provided 36 times as much DHA $(120 \mathrm{mg} / \mathrm{d}$ versus 1.7 or $4.3 \mathrm{~g} / \mathrm{d}$, respectively)? Since different tests of cognitive function were employed and the supplements were given for different periods, were these interventions studies actually comparable? As implied in the study by Freund-Levi et al. [10], are those with higher cognitive function at the start of the trial actually likely to benefit more from a $\omega 3$ supplement? Are those with lower cognitive function actually at greater risk when given a $\omega 3$ supplement?

\section{Brain levels of DHA in Alzheimer's disease}

If $D H A$ supplementation is beneficial in $A D$ or $\mathrm{MCl}$, it seems reasonable to expect that this benefit relates to correcting lower brain DHA. Although we have found no reports of brain DHA levels in humans after DHA supplementation (in $A D$ or otherwise), we are aware of the following reports on brain $\mathrm{DHA}$ content in $\mathrm{AD}$ :
Bowen et al. [15] appear to be the first to have reported brain fatty acid data in AD. They analyzed fatty acid profiles of phospholipids from frontal grey matter from controls $(n=12)$, senile dementia $(n=11), A D(n=4)$ and Creuzfeldt-Jacob disease $(n=1)$. Their method pre-dated capillary gas chromatography so did not permit good separation of specific polyunsaturates. As a result of this important limitation, AA was grouped with adrenic acid (22:4ஸ6) and DHA was grouped with the two docosapentaenoic acids. These data were reported as 'polyunsaturates'. They found no age- or disease-related change in brain polyunsaturates or phospholipid concentration.

In 1991, Soderberg et al. published the most widely cited paper on fatty acid composition of brain samples obtained at autopsy from 8-10 controls and AD patients [16]. Brain samples were analyzed from four different regions - frontal grey matter, white matter (region not specified), hippocampus and pons. Fatty acids were analyzed from two of the main classes of phospholipids in the brain - phosphatidylethanolamine (PE) and phosphatidylcholine, which are rich and poor, respectively, in polyunsaturated fatty acids. The $\%$ of DHA in PE was reduced by up to $50 \%$ in all four brain regions from patients with $A D$. The fatty acid changes attributed to $A D$ were not be due to aging alone which, as they reported in the same paper, was not associated with changes in brain fatty acid composition.

In 1993, Skinner et al. [17] reported brain fatty acid composition in $15 \mathrm{AD}$ patients and 10 controls. Grey and white matter of frontal, parietal and parahippocampal regions were studied. Amongst the polyunsaturates, there were no differences due to $A D$ except for 3-4 fold higher levels of adrenic acid in grey matter, as well as higher DHA in parietal white matter.

In 1998, Corrigan et al. [18] reported the fatty acid composition of parahippocampal cortex in $8 \mathrm{AD}$ patients and 6 age-matched controls. $A D$ was confirmed histologically in each case. DHA was not different between $A D$ and controls for any of the four phospholipid classes or in the cholesteryl ester class. In the AD samples, adrenic acid was lower in both PE and phosphatidylserine, and AA was also lower in PE. In the cholesteryl esters of AD brain, $\alpha$-linolenic acid $(18: 303)$ was present at only $5 \%$ of the level seen in controls.

In 1998, Prasad et al. [19] evaluated brain fatty acid composition in $9 \mathrm{AD}$ patients and 9 agematched controls. Fatty acids were quantified $(\mathrm{nmol} / \mathrm{g})$ in several brain regions and in three phospholipid classes as well as in brain free fatty acids. DHA was $45 \%$ lower in PE from the hippocampus and parahippocampal gyrus (both regions analyzed together), and was 
$24 \%$ lower in the phosphatidylcholine fraction of the cerebellum. Lower DHA in hippocampal and parahippocampal PE was actually due to lower PE because, on a percentage basis, DHA was $50 \%$ higher in this lipid class. Otherwise, DHA did not differ across lipid fractions or brain regions. This study emphasized the possible importance of lower plasmalogens, a form of $P E$ with vinyl-linked instead of ester-linked fatty acids, in the progression of $A D$. In AD, a lower concentration plasmalogens was observed but only in the hippocampus [19].

In 1999, Guan et al. [20] reported the phospholipid and fatty acid composition of brain samples from $A D$ patients $(n=15 ; 80$ y old $)$ and controls $(n=13 ; 72$ y old). Data were provided for frontal cortex, hippocampus and white matter and were expressed relative to brain DNA. In the AD samples, the content of the two main phospholipids was $16-24 \%$ lower in the frontal cortex, an effect due primarily to lower plasmalogen PE. Expressed in relation to brain DNA content, DHA was not different in the various lipid classes of frontal cortex from $\mathrm{AD}$ patients compared to the controls. Given that the concentration of plasmalogen PE in the frontal cortex was decreased in $A D$ brain but the DHA concentration in plasmalogen PE was unchanged, the $\%$ of DHA in this lipid class actually increased somewhat. In contrast, AA was decreased by $49 \%$ and adrenic acid was decreased by $27 \%$ in the plasmalogen PE of frontal cortex from $A D$ patients. Oleic acid $(18: 1 \omega 9)$ was also decreased by $25-75 \%$ in the same regions, an effect not reported elsewhere. In 2001, Han et al. [21] reported the fatty acid composition of the various molecular species of $P E$ in several brain regions from $A D$ patients who were separated according to different levels of cognitive decline $(0.5$ to 3 on the clinical dementia rating scale. There were 6 samples in the control group and in each of the four grades of AD. They showed that even at the earliest clinically defined stage of $A D$, plasmalogen PE was decreased by up to $40 \%$ in white matter. In gray matter, this decline in PE was less severe but also correlated with clinical severity. DHA concentrations (mg/g protein) were lower in some brain regions as were other fatty acids, including saturates, monounsaturates and $\omega 6$ polyunsaturates. The only brain region in which lower DHA was not due to lower PE was in the cerebellum.

Taken collectively, published brain DHA data are available from about $85 \mathrm{AD}$ patients and 54 controls but it is impossible to draw any clear conclusion about a direct link between lower brain $\mathrm{DHA}$ and $\mathrm{AD}$. When low DHA was observed, it was not specific for DHA: either AA and/or adrenic acid were also decreased [16, $18,21]$, or the lower DHA was a function of a

decrease in one of the lipid classes, most com- monly plasmalogen PE [20]. Increases of up to $50 \%$ for DHA $[17,19,20]$ and 4 fold for adrenic acid [17] have also been reported for some regions of $A D$ brain. Hence, it is clearly incorrect to discuss lower brain DHA as if it were a commonly accepted or specific observation in $A D$. These studies actually seem to agree more about changes in brain phospholipids in $A D$, particularly the decrease in plasmalogen $P E$, which may be equally or more important as the possible changes in their fatty acid composition [22]. Therefore, the comment made by Guan et al. [20] nearly a decade ago still seems highly pertinent - 'these discrepancies are largely due to diagnostic problems, methodological differences as well as to calculation and presentation'.

Good data from age-matched controls are of critical importance to interpreting changes in brain lipid or fatty acid composition attributable to AD. Svennerholm et al. [23] reported age stratified data for brain composition from people living at home who experienced accidental deaths. They had 40-50 samples per age group and showed that between the lowest age group (20-39y) and the highest (80-100 y), brain weights decreased by $20 \%$ and brain phospholipid content decreased by about $43 \%$. Nevertheless, no evaluation of cognitive function or fatty acid composition was reported. Soderberg et al. [16] reported age-stratified data showing that aging alone does not change brain fatty acid in either of the two main phospholipid classes of the brain.

Therefore, agreement is lacking on even the basic point of whether healthy aging is associated with a change in brain fatty acid composition $[16,23]$, so there is a real possibility that some of the discrepancies regarding whether or not brain DHA changes in AD may actually be due to difficulty in selecting healthy, agematched controls or in classifying the $A D$ patients themselves. Subcellular changes in DHA should be explored to see whether they are partially responsible for the wide disparities in reported effects of AD on brain DHA. The reports of lower brain $A A$ and adrenic acid in $A D$ should also not be overlooked because $A A$ may have clinically relevant effects on cognition [13].

Table 1. Strengthening the putative link between low docosahexaenoic acid (DHA) and Alzheimer's disease will require resolution of the following issues.

1. Do the healthy elderly metabolize $\omega 3$ fatty acids differently than healthy young adults? 2. Does the brain of the healthy elderly contain less DHA than in healthy young adults? 3. In adult humans, is brain DHA content linked to DHA intake?

4. Is the effect of a DHA supplement on performance of cognitive tests dose dependant? 5. Does the dose of DHA affecting performance on cognitive tests depend on background DHA intake? 6. Do other $\omega 3$ or $\omega 6$ polyunsaturates contribute to the mild beneficial effect on cognition presently attributed to DHA?

\section{Oversimplification of the relation between DHA and Alzheimer's disease?}

Assuming that low fish intake does in fact increase the risk of $A D$, should low brain $D H A$ be necessarily expected in $A D$ or, indeed, that DHA would be clinically effective in AD? DHA synthesis and $\beta$-oxidation are both very low in humans $[24,25]$. Therefore, unlike in many animal models, in humans, DHA intake does have an important effect on whole body DHA homeostasis [26]. Nevertheless, dietary $\omega 3$ fatty acid depletion studies show that relative slower to deplete or to replete after dietary depletion. Hence, in humans, the link between DHA intake and brain DHA is potentially complex and it is difficult to make the case that there should necessarily be a direct or simple link between low DHA intake, low plasma DHA and low brain DHA in humans, let alone in $A D$. Especially in the elderly, factors linked to DHA degradation (peroxidation or loss due to cell death) in the brain may well be as important in determining brain DHA levels as the input variables such as dietary supply of DHA, a trickle of synthesis, or its transport into the brain. Neverment for preventing or treating $A D$ depends to some extent on understanding how DHA is utilized by the aging brain (table 1), so as to establish when its use is to be encouraged and when it is contraindicated.

$\mathrm{AD}$ has a major impact on the healthcare delivery system and has no widely accepted treatment. Exploration of strategies to reduce its risk and possibly improve its treatment is therefore an urgent matter. Notwithstanding the reservations we express here about the lack of support from analysis of brain DHA and weak support from clinical trials, DHA still stands to apparent association between low DHA intake and increased risk of cognitive decline is really to bear fruit, several issues need to be kept in focus. Most importantly and as previously proposed [27, 28], well designed clinical trials are needed; plausibility and enthusiasm alone cannot supplant this crucial information. to DHA in blood or other tissues, brain DHA is theless, the optimization of DHA as a supplebe a key component of such strategies. If the 
Other issues to be resolved include different definitions of cognitive function, different time courses and durations of treatment, and potential benefits as well as hazards of isolated nutrients versus foods, i.e. DHA supplements versus higher intake of fish and seafood. Protective effects against all causes of dementia of as little as $180 \mathrm{mg} / \mathrm{d}$ of DHA have been proposed [7], yet this amount is $10 \%$ or less of the DHA dose used in two clinical studies [10,12], and is also well below even the background DHA intake in the latter studies ( $>500 \mathrm{mg} / \mathrm{d}$ ). Very low background DHA intakes, i.e. in the USA, could hypothetically reduce the dose of DHA needed to have a clinical benefit, but this still needs to be established.

Are higher cognitive scores predictive of a better DHA effect? Oxidative stress is believed to play a role in $A D$ so supplementation with a highly peroxidizable fatty acid like DHA may be contraindicated in some cases, i.e. perhaps when the disease is more advanced. Lower brain $A A$ has been reported in $A D$ and may contribute to the very mild clinical improvement reported in $\mathrm{MCl}$, so should $\mathrm{AA}$ or other $\omega 6$ fatty acids $[13,29]$ be evaluated as a treatment for $\mathrm{MCl}$ or $\mathrm{AD}$ ? EPA is normally virtually undetectable in the human brain but it may still have contributed to the mild beneficial effect on $A D$ symptoms attributed to DHA [10-12]. Perhaps further attention should be paid to the clinical efficacy in $\mathrm{AD}$ or $\mathrm{MCl}$ of pure EPA [14].

Finally, we present preliminary data here suggesting that short-term plasma DHA response to a DHA supplement is significantly higher in the healthy elderly than in healthy young adults (figure 1). Much more therefore need to be done to establish whether healthy aging changes $\omega 3$ fatty acid metabolism; such data will be needed to better interpret DHA effects in disease states affecting the elderly, especially AD. Indeed, DHA or other $\omega 3$ polyunsaturates are not the only components of fish that could be responsible for protecting the brain against cognitive deterioration - marine foods are rich in other nutrients such as iodine, which may contribute towards maintaining cognitive function in the elderly [30].

Acknowledgements. Financial support for our research contributions in this field was provided by the Canada Research Chairs program (SCC), Canadian Foundation for Innovation, Canadian Institutes for Health Research, Natural Sciences and Engineering Research Council, AFMNet, Fonds de recherche en Santé du Québec, Health and Social Sciences Center - University Institute of Geriatrics of Sherbrooke, the Research Center on Aging, the Department of Medicine, Université de Sherbrooke (fellowship to MP),

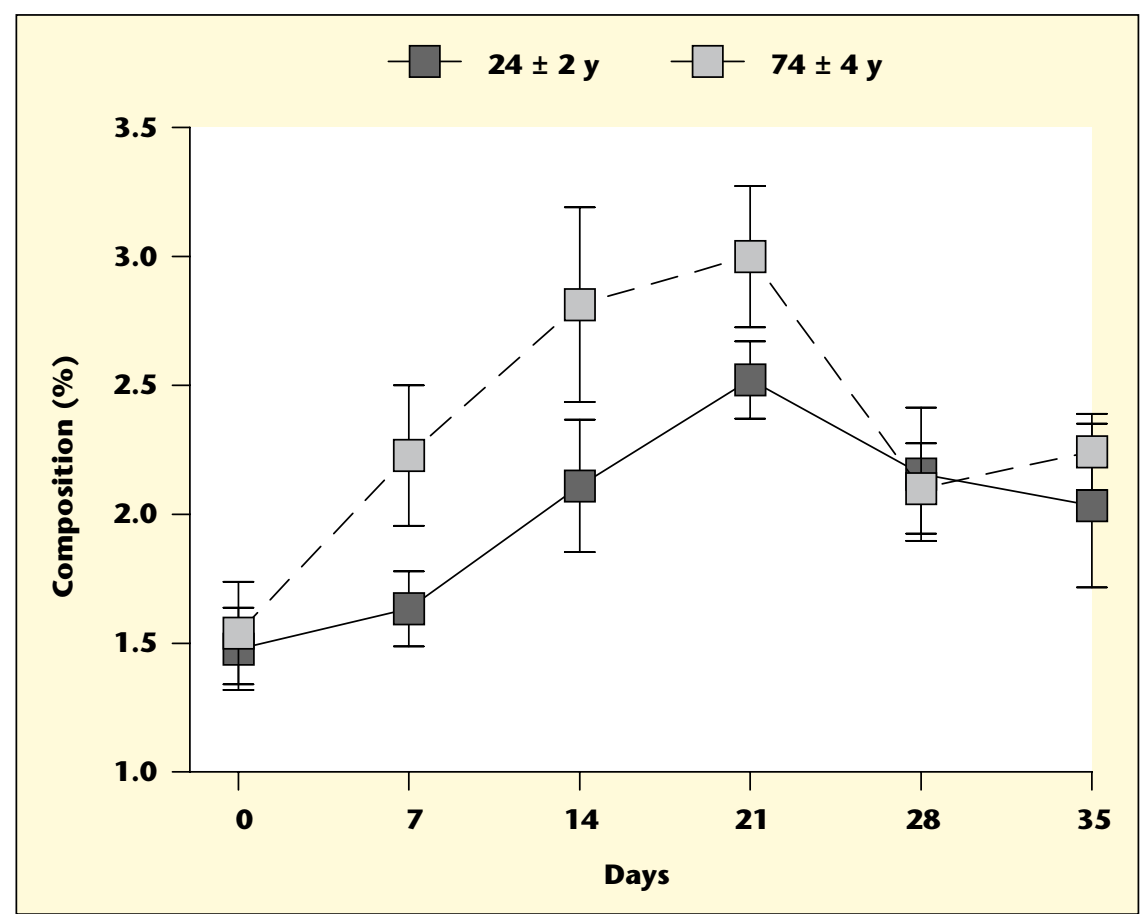

Figure 1. Changes in the \% composition of docosahexaenoic acid (DHA) in plasma total lipids during 21 days supplementation with $680 \mathrm{mg} / \mathrm{d}$ of (DHA) in elderly versus young adults (74 \pm 4 versus $24 \pm 2$ y old, respectively; $n=9-10$ (group). The supplementation period was followed by a 14 day wash-out. Although starting DHA values were similar and the slopes of the rise and fall of plasma DHA did not differ between the two groups, at d 21, plasma $(\mathrm{DHA})$ was $42 \%$ higher in the elderly $(p<0.05)$. Compliance was independently verified by adding carbon- $13\left({ }^{13} \mathrm{C}\right)$ glucose to the $\mathrm{DHA}$ capsules and measuring ${ }^{13} \mathrm{C}$ in breath $\mathrm{CO}_{2} 2 \mathrm{~h}$ after the capsules were consumed. Participants were blinded to the presence of the ${ }^{13} \mathrm{C}$-glucose on the capsules and to the reason for breath sampling. This study suggests that the healthy elderly have a higher short-term plasma response to dietary DHA than do healthy young adults, an effect that is independent of compliance.

Réseau Québecois de recherche sur le vieillissement, and FORMSAV (studentship to MV). Mary Ann Ryan, Dr. Sébastien Tremblay and Julie Desgagné provided excellent technical support.

\section{REFERENCES}

1. SOBOCKIP, JÖNSSON B. Wittchen HansUlrich, Olesen J. Cost of disorders of the brain in Europe. Eur / Neurol 2005; 12(suppl 1): 1-27.

2. GILLETTE GUYONNETS, ABELLAN VAN KAN G, ANDRIEU $S$, et al. IANA task force on nutrition and cognitive decline with aging. J Nutr Health 2007; 11(2): 132-52.

3. BOURRE JM. Roles of unsaturated fatty acids (especially omega-3 fatty acids) in the brain at various ages and during ageing. I Nutr Health Aging 2004; 8(3): 163-74.

4. VAN GELDER BM, TIJHUIS M, KALMIIN S, KROMHOUT D. Fish consumption, n-3 fatty acids, and subsequent 5 -y cognitive decline in elderly men: the Zutphen Elderly Study. Am J Clin Nutr 2007; 85(4): 1142-7.

5. BEYDOUN MA, KAUFMAN IS, SATIA JA, ROSAMOND W, FOLSOM AR. Plasma n-3 fatty acids and the risk of cognitive decline in older adults: the Atherosclerosis Risk in Communities Study. Am / Clin Nutr 2007; 85(4): 1103-11.
6. CONQUER JA, TIERNEY MC, ZECEVIC I, BETTGER W], FISHER RH. Fatty acid analysis of blood plasma of patients with Alzheimer's disease, other types of dementia, and cognitive impairment. Lipids 2000; 35(12): 1305-12.

7. SCHAEFER EJ, BONGARD V, BEISER AS, et al. Plasma phosphatidylcholine docosahexaenoic acid content and risk of dementia and Alzheimer disease: the Framingham Heart Study. Arch Neurol 2006; 63(11): 1545-50.

8. TULLY AM, ROCHE HM, DOYLE R, et al. LOW serum cholesteryl ester-docosahexaenoic acid levels in Alzheimer's disease: a case-control study. Br / Nutr 2003; 89(4): 483-9.

9. LAURIN D, VERREAULT R, LINDSAY J, DEWAILLY E, HOLUB B]. Omega-3 fatty acids and risk of cognitive impairment and dementia. / Alzheimers Dis 2003; 5(4): 315-22.

10. FREUND-LEVI Y, ERIKSDOTTER-JONHAGEN M, CEDERHOLM T, et al. Omega-3 fatty acid treatment in 174 patients with mild to moderate Alzheimer disease: OmegAD study: a randomized double-blind trial. Arch Neurol 2006; 63(10): 1402-8 
11. TERANO T, FUIISHIRO S, BAN T, et al. Docosahexaenoic acid supplementation improves the moderately severe dementia from thrombotic cerebrovascular diseases. Lipids 1999; 34(Suppl): S345-S346.

12. SUZUKI H, MORIKAWA Y, TAKAHASHI H. Effect of DHA oil supplementation on intelligence and visual acuity in the elderly. World Rev Nutr Diet 2001; 88: 68-71.

13. KOTANIS, SAKAGUCHIE, WARASHINA $S$ et al. Dietary supplementation of arachidonic and docosahexaenoic acids improves cognitive dysfunction. Neurosci Res 2006; 56(2): 159-64

14. BOSTON PF, BENNETT A, HORROBIN DF, BENNETT CN. Ethyl-EPA in Alzheimer's disease-a pilot study. Prostaglandins Leukot Essent Fatty Acids 2004; 71(5): 341-6.

15. BOWEN DM, SMITH CB, DAVISON AN. Molecular changes in senile dementia. Brain 1973; 96(4): 849-56.

16. SODERBERG M, EDLUND C, KRISTENSSON K, DALLNER G. Fatty acid composition of brain phospholipids in aging and in Alzheimer's disease. Lipids 1991; 26(6): 421-5.

17. SKINNER ER, WATT C, BESSON JA, BEST PV. Differences in the fatty acid composition of the grey and white matter of different regions of the brains of patients with Alzheimer's disease and control subjects. Brain 1993; 116(Pt 3): 717-25.
18. CORRIGAN FM, HORROBIN DF, SKINNER ER, BESSON JA, COOPER MB. Abnormal content of $n-6$ and n-3 long-chain unsaturated fatty acids in the phosphoglycerides and cholestero esters of parahippocampal cortex from Alzheimer's disease patients and its relationship to acetyl CoA content. Int I Biochem Cell Biol 1998; 30(2): 197-207.

19. PRASAD MR, LOVELL MA, YATIN M, DHILLON H, MARKESBERY WR. Regional membrane phospholipid alterations in Alzheimer's disease. Neurochem Res 1998; 23(1): 81-8.

20. GUAN Z, WANG Y, CAIRNS NJ, LANTOS PL, DALLNER G, SINDELAR PJ. Decrease and structural modifications of phosphatidylethanolamine plasmalogen in the brain with Alzheimer disease. J Neuropathol Exp Neurol 1999; 58(7): 740-7.

21. HAN X, HOLTZMAN DM, MCKEEL JR. DW. Plasmalogen deficiency in early Alzheimer's disease subjects and in animal models: molecular characterization using electrospray ionization mass spectrometry. J Neurochem 2001; 77(4): 1168-80.

22. FAROOQUI AA, HORROCKS LA, FAROOQUI T. Modulation of inflammation in brain: a matter of fat. J Neurochem 2007; 101(3): 577-99.

23. SVENNERHOLM L, BOSTROM K, JUNGBJER B. Changes in weight and compositions of major membrane components of human brain during the span of adult human life of Swedes. Acta Neuropathol (Berl) 1997; 94(4): 345-52.
24. FREEMANTLE E, VANDAL M, TREMBLAYMERCIER J, et al. Omega-3 fatty acids, energy substrates, and brain function during aging. Prostaglandins Leukot Essent Fatty Acids 2006; 75(3): 213-20.

25. PLOURDE M, CUNNANE SC. Extremely limited synthesis of long chain polyunsaturates in adults: Implications for their dietary essentiality and use as supplements. Appl Physiol Nutr Metab 2007; (in press).

26. ARTERBURN LM, HALL EB, OKEN H. Distribution, interconversion and dose response of $n-3$ fatty acids in humans. Am / Clin Nutr 83 (suppl): 14675-765

27. ISSA AM, MOJICA WA, MORTON SC, et al. The efficacy of omega-3 fatty acids on cognitive function in aging and dementia: a systematic review. Dement Geriatr Cogn Disord 2006; 21(2): 88-96.

28. MACLEAN CH, ISSA AM, NEWBERRY SJ, et al. Effects of omega- 3 fatty acids on cognitive function with aging, dementia, and neurological diseases. Evid Rep Technol Assess (Summ) 2005(114): 1-3.

29. CORRIGAN FM, VAN RHIJN A, HORROBIN DF. Essential fatty acids in Alzheimer's disease. Ann N Y Acad Sci 1991; 640: 250-2.

30. BÉGIN ME, LANGLOIS MF, LORRAIN D, CUNNANE SC. Thyroid function and cognition during aging. Handbook of lodine. 2007; (in press). 